\title{
Malignant Deciduoid Mesothelioma: case presentation of an exceptional variant and review of the literature
}

\author{
Mouna Khmou ${ }^{1,2^{*}}$, Soumiya Echcharif1,2, Rachad Kabbaj ${ }^{1,2}$ and Basma El Khannoussi ${ }^{1,2}$
}

\begin{abstract}
Background: Malignant Deciduoid Mesothelioma (MDM) is an extremely rare variant of epithelioid mesothelioma. It was first described in young females, in the peritoneum, and its relation with asbestos was not well defined. Later reports, have shown that this variant may also occur in the pleura, the pericardium and the tunica vaginalis of elderly people, who had been exposed to asbestos.

Case presentation: We report a case of malignant deciduoid mesothelioma that occurred in the peritoneal cavity, and the omentum of a 35-year-old woman. The patient had never been exposed to asbestos.

Conclusions: Through this observation, we describe clinical, histopathological, and immunohistochemical findings of deciduoid mesothelioma, and review the literature reports.
\end{abstract}

Keywords: Mesothelioma, Deciduoid, Peritoneum, Immunohistochemistry

\section{Background}

MDM is an extremely rare subtype of malignant epithelioid mesothelioma, with a poor prognosis. It was first described by Talerman et al. [1] in 1985 and Nascimento et al. [2] in 1994, characterized by the presence of cytomorphological features resembling decidual reaction [3].

Few cases have been reported in the literature to date, in the form of single case reports. A total of 25 cases of deciduoid peritoneal mesothelioma have been documented in the English literature, since this entity was first described in 1985 [1, 2, 4-17].

The aim of the present report is to describe MDM of the peritoneum, to discuss the differential diagnosis and management of this extremely rare tumour.

\section{Case presentation}

A 35-year-old female patient, with no medical history, was admitted for an appendicular syndrome. There was no history of asbestos exposure. On physical examination, she had a low-grade fever. A generalized abdominal tenderness and distension were also reported.

\footnotetext{
* Correspondence: mouna.khmou@yahoo.fr

${ }^{1}$ Department of Pathology, National Institute of Oncology, Rabat, Morocco

${ }^{2}$ Faculty of Medicine and Pharmacy Rabat, University Mohammed V, Rabat, Morocco
}

Abdominal ultrasound examination showed moderate gray, infiltrating the peritoneal cavity, and the omentum. A biopsy was performed. We received four firm, gray white fragments. Microscopically, H\&E stain showed sheets of malignant cells, formed exclusively by large pleomorphic epithelioid cells with abundant, eosinophilic cytoplasm. The nuclei were large and vesicular, with prominent nucleoli (Figs. 1 and 2). The cell borders were variably well defined. Focally tumor cells showed vacuolated cytoplasm, with binucleated and multiLymphoplasmacytic and neutrophilic infiltration was In immunohistochemistry, tumor cells were positive for cytokeratin AE1/AE3, calretinin (Fig. 3), D2-40, CK5/6 and WT-1. They were negative for Ber-EP4, $\mathrm{CEA}$, estrogen (ER) and progesterone (PR) receptors, and HMB-45. The microscopic and immunohistochemical findings were compatible with deciduoid malignant mesothelioma. The patient received 5 cycles of chemotherapy. She presently has a survival of 12 months. 


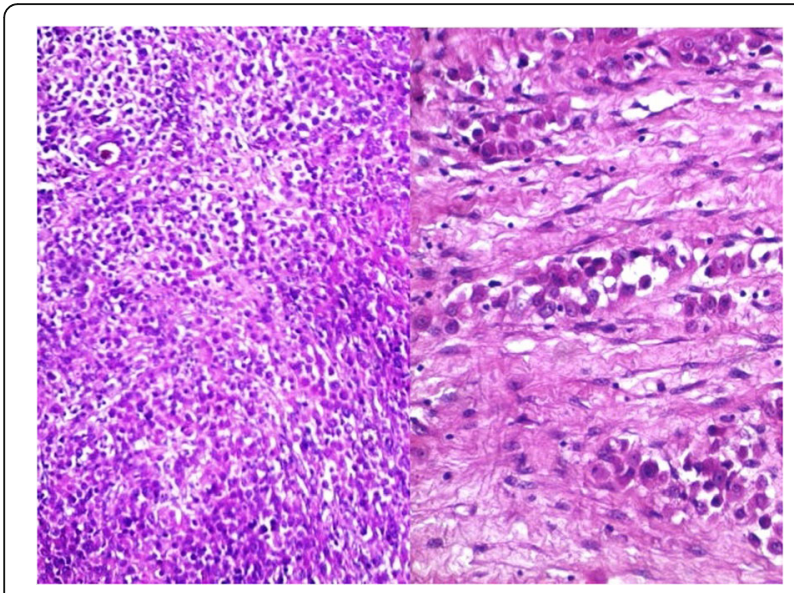

Fig. 1 Histological section showing large solid sheets of malignant epithelioid cells

\section{Discussion}

Deciduoid mesothelioma was initially reported in the peritoneum of young women, with no history of asbestos exposure. Later reports have demonstrated that this entity could occur in the pleura and pericardium of older men and women who had been exposed to asbestos $[15,16]$.

This designation was first introduced by Nascimento et al. [2] in 1994, to describe a rare variant of epithelioid mesothelioma, that reflect a morphological resemblance with decidua or decidual type changes [15].

The scarcity of this entity was highlighted by Ordonez and al. [15] in the latest review of malignant deciduoid mesothelioma. Only 25 cases of deciduoid peritoneal mesothelioma have been reported until today [1, 2, 4-17]. Seventeen of these patients were female, with ages ranging from 8 to 78 years. Nineteen cases were unrelated etiologically to asbestos. The five cases associated with asbestos exposure were noted in elderly patients (Table 1).

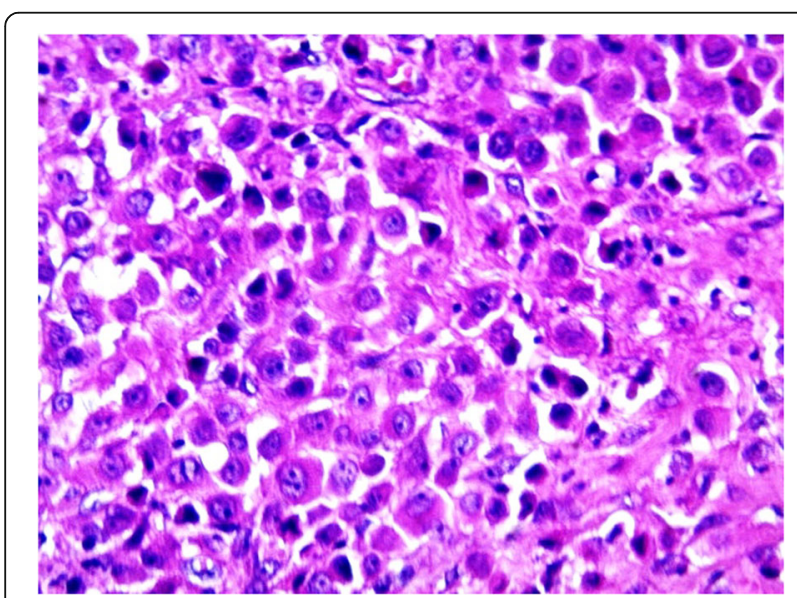

Fig. 2 Decidual tumor cells with large eosinophilic cytoplasm and prominent nucleoli

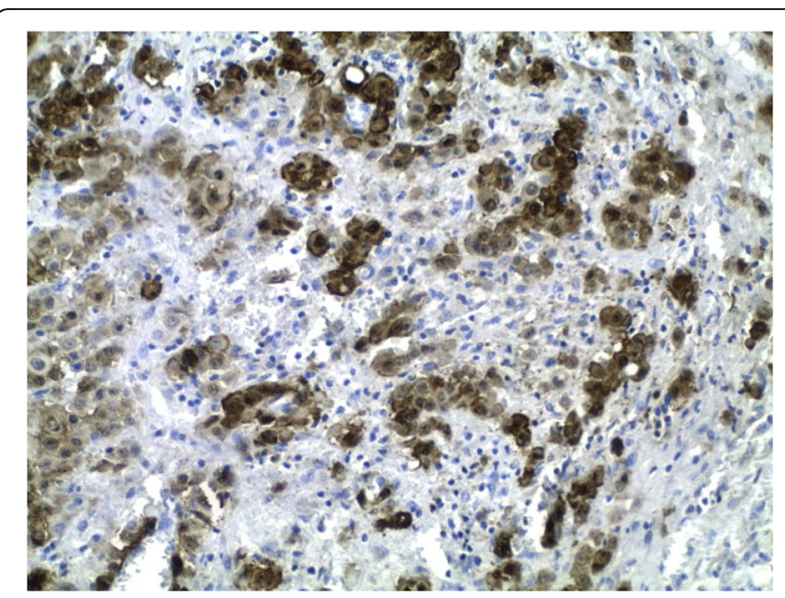

Fig. 3 tumor cells with positive immunoreactivity to calretinin

Clinically, symptoms are often insidious and nonspecific, causing a delay in diagnosis; the imaging findings are also not specific. Histologically, there are four major subtypes of mesothelioma: epithelioid, sarcomatoid, desmoplastic and biphasic. Deciduoide mesothelioma is a rare subtype of epithelioid mesothelioma, in which a wide range of histopathological patterns is described [18, 19].

Microscopically, deciduoid mesothelioma is composed of large, polygonal, or round cells, arranged in solid nests and in trabecula $[13,18,19]$. The cytoplasm is abundant eosinophilic with well-defined cell borders $[18,19]$. The nuclei is large and vesicular, with prominent nucleoli. $\mathrm{Nu}$ clear pleomorphism, nuclear pseudoinclusions, multinucleated cells have been reported. Mitotic index is variable, with high mitotic activity and atypical mitoses observed in some cases. Several features described in the literature include the presence of foamy cells, with clear cytoplasm, and mucinous or myxoid stroma [15].

A large immunohistochemical panel is essential for accurate diagnosis, and help in distinguishing differentiel diagnosis. The International Mesothelioma Panel and the WHO recommend to use at least 2 mesothelial and 2 carcinoma markers, in addition to a pancytokeratin $[19,20]$. The choice of immunohistochemical markers used varies depending on the histologic type of mesothelioma, the location of the tumor (pleural versus peritoneal), and the type of tumor being considered in the differential diagnosis [20].

Based on their specificity and sensitivity, calretinin, CK5/6, EMA, WT-1 and D2-40 are de the best mesothelial markers, and are positive in MDM [19]. Ber-EP4, B72.3, and carcinoembryonic antigen are not expressed in mesothelioma. They are the most commonly used markers to eliminate carcinoma (primary peritoneal carcinoma, and secondary peritoneal carcinomatosis) [19]. Others markers can be added to assist in the differential diagnosis. 
Table 1 Summary of the cases of Malignant Deciduoid Mesothelioma in the peritoneum, reported in the literature

\begin{tabular}{|c|c|c|c|c|}
\hline Author(s) & Sex/age & Asbestos exposure & Type of specimen & Histology \\
\hline Talerman et al. [1] & $F / 13$ & No & Biopsy & Deciduoid \\
\hline \multirow[t]{2}{*}{ Nacimento et al. [2] } & $F / 23$ & No & Biopsy & Deciduoid \\
\hline & $F / 24$ & No & Biopsy & Deciduoid \\
\hline Orosz et al. [4] & $F / 15$ & No & Biopsy & Deciduoid \\
\hline \multirow[t]{4}{*}{ Shanks et al. [5] } & $F / 53$ & Yes & Biopsy & Deciduoid \\
\hline & $M / 65$ & Yes & Biopsy + autopsy & Deciduoid \\
\hline & $M / 55$ & Yes & Tumor resection & Deciduoid + tubular and sarcomatoid areas \\
\hline & $\mathrm{F} / 55$ & No & Biospy & $\begin{array}{l}\text { Predominantly tubulopapillary + } \\
\text { sarcomatoid areas, } \\
\text { deciduoid cells }\end{array}$ \\
\hline Desai et al. [6] & $F / 53$ & NA & Tumor resection & Deciduoid \\
\hline Gillespie et al. [7] & $\mathrm{F} / 50$ & No & Peritoneal cytology & Deciduoid \\
\hline Okonkwo et al. [8] & $F / 30$ & No & Tumor resection & Deciduoid \\
\hline \multirow[t]{2}{*}{ Shia et al. [9] } & $F / 51$ & NA & Tumor resection & Deciduoid + tubulopapillary \\
\hline & $M / 56$ & Yes & Resected specimen & Deciduoid + tubulopapillary \\
\hline Chung et al. [10] & $F / 47$ & No & Resected tumor & Deciduoid \\
\hline Maeda et al. [11] & $F / 24$ & No & Resected tumor & Deciduoid \\
\hline Kimura et al. [12] & M/70 & No & $\begin{array}{l}\text { Peritoneal biopsy, } \\
\text { autopsy }\end{array}$ & Deciduoid \\
\hline Mourra et al. [13] & $F / 41$ & No & Tumor resection & Deciduoid with pleomorphic nuclei \\
\hline Ustun et al. [14] & $F / 59$ & No & Tumor resection & Deciduoid \\
\hline \multirow[t]{4}{*}{ Ordonez [15] } & M/78 & No & Biopsy & Deciduoid \\
\hline & $M / 62$ & No & Peritoneal nodules & Deciduoid \\
\hline & $M / 56$ & No & Biopsy & Deciduoid \\
\hline & $F / 39$ & No & Biopsy & Deciduoid \\
\hline \multirow[t]{2}{*}{ Huang [16] } & $M / 18$ & No & Biopsy & Deciduoid \\
\hline & $F / 64$ & Yes & Biopsy & Deciduoid \\
\hline Wolff-bar [17] & $F / 8$ & No & Cytoreductive surgery & Deciduoid + tubulopapillary \\
\hline Our case & $F / 35$ & No & Biopsy & Deciduoid \\
\hline
\end{tabular}

NA Not Available

Our findings illustrated that calretinin, AE1/AE3, CK5/6, D2-40 and WT-1 were positive. Ber-EP4, CEA showed a negative result, respectively, which is consistent with the literature.

Peritoneal Deciduoid mesotheliomas of women should be distinguished from diffuse pseudotumoral deciduosis. This condition has been frequently associated with pregnancy, but also observed in the perimenarchal and postmenopausal periods [15]. Microscopically, the nuclei are smaller and have dark dense chromatin [13]. Decidual cells lack expression of pancytokeratin and calretinin. However, they strongly express CD10, alpha inhibin, estrogen and progesterone receptors $[15,21]$.

Trophoblastic neoplasia is also important to consider in the differential diagnosis [21]. The neoplastic cells in this condition express HCG [15].
Positive staining for AE1/AE3 and calretinin are useful to exclude Rhabdomyosarcoma, epithelioid gastrointestinal stromal tumor (GIST), melanoma and epithelioid angiosarcoma [16, 21]. Immunohistochemical staining should easily distinguish these entities, using Desmin and myogenin for rhabdomyosarcoma; C-kit and Dog1 for GIST; Melan-A, HMB-45 for melanoma; and ERG for angiosarcoma [21].

Chemotherapy and cytoreductive surgery are usually the best therapeutic options. Cisplatin and pemetrexed have demonstrated a significant survival advantage, and are considered as firstline treatment $[16,17]$.

The deciduoid subtype was regarded by some authors to be a more aggressive variant [6]. However, some recent reviews of reported cases did not reveal a significantly worse survival in patients with deciduoid features. 
There are no large survival studies for MDM due to its rarity [9].

\section{Conclusions}

In summary, through this observation, we want to highlight the rarity of this variant. It is important for pathologists to recognize this morphological variant of epithelioid mesothelioma, easily confused with other diangnosis, and discuss the available treatment options. We believe that more studies on a larger number of cases may be helpful in further defining this entity.

\section{Abbreviations}

ER: estrogen receptors; H\&E: Hematoxylin and eosin; MDM: Malignant Deciduoid Mesothelioma; PR: progesterone receptors; WHO: World Health Organization

\section{Acknowledgements \\ None. \\ Funding \\ None.}

\section{Availability of data and materials}

All data generated or analysed during this case are included in this published article.

\section{Authors' contributions \\ MK analyzed and interpreted the patient data, drafted the manuscript and made the figures. BE performed the histological examination, proposed the study, supervised MK and revised the manuscript. SE and RK have made substantial contributions to analysis and interpretation of patient data. All authors read and approved the final manuscript.}

\section{Ethics approval and consent to participate}

Not applicable.

\section{Consent for publication}

Written informed consent was obtained from the patient for publication of this Case Report and any accompanying images. A copy of the written consent is available for review by the Editor-in-Chief of this journal.

\section{Competing interests}

The authors declare that they have no competing interests.

\section{Publisher's Note}

Springer Nature remains neutral with regard to jurisdictional claims in published maps and institutional affiliations.

Received: 6 April 2017 Accepted: 11 August 2017

Published online: 18 August 2017

\section{References}

1. Talerman A, Montero JR, Chilcote RR, Okagaki T. Diffuse malignant peritoneal mesothelioma in a 13-year-old girl. Report of a case and review of the literature. Am J Surg Pathol. 1985;9:73-80.

2. Nascimento AG, Keeney GL, Fletcher CD. Deciduoid peritoneal mesothelioma. An unusual phenotype affecting young females. Am I Surg Pathol. 1994;18:439-45.

3. Santosa C, Gamboaa F, Fradinhoa F, Pêgoa A, Carvalhob L. Bernardoc J Deciduoid pleural mesothelioma - a rare entity in a young woman. Rev Port Pneumol. 2012;18(6):294-8.

4. Orosz Z, Nagy P, Szentirmay Z, et al. Epithelial mesothelioma with deciduoid features. Virchows Arch. 1999:434:263-6.

5. Shanks JH, Harris M, Banerjee SS, et al. Mesotheliomas with deciduoid morphology: a morphologic spectrum and a variant not confined to young females. Am J Surg Pathol. 2000;24:285-94.
6. Desai S, Kane S, Bharde S, et al. Malignant peritoneal mesothelioma deciduoid or anaplastic variant? Point to ponder. Indian J Pathol Microbiol. 2001;44:159-62.

7. Gillespie FR, van derWalt JD, Derias N, et al. Deciduoid peritoneal mesothelioma. Rep Cytological Appearances Cytopathol. 2001;12:57-61.

8. Okonkwo A, Musunuri S, Diaz L Jr, et al. Deciduoid mesothelioma: a rare, distinct entity with unusual features. Ann Diagn Pathol. 2001;5:168-71.

9. Shia J, Erlandson RA, Klimstra DS. Deciduoid Mesothelioma: a report of 5 cases and literature review. Ultrastruct Pathol. 2002;26:355-63.

10. Chung DJ, Kang YW, Kim BK, et al. Deciduoid peritoneal mesothelioma: CT findings with pathologic correlation. Abdom Imaging. 2003;28:614-6.

11. Maeda S, Hosone M, Katayama H, et al. Deciduoid mesothelioma in the pelvic cavity. Pathol Int. 2004;54:67-72.

12. Kimura N, Ogasawara T, Asonuma S, et al. Granulocyte-colony stimulating factor- and interleukin 6- producing diffuse deciduoid peritoneal mesothelioma. Mod Pathol. 2005;18:446-50.

13. Mourra N, de Chaisemartin C, Goubin-Versini I, et al. Malignant deciduoid mesothelioma: a diagnostic challenge. Arch Pathol Lab Med. 2005;129:403-6.

14. Ustun H, Astarci HM, Sungu N, et al. Primary malignant deciduoid peritoneal mesothelioma: a report of the cytohistological and immunohistochemical appearances. Diagn Cytopathol. 2011;39:402-8.

15. Ordonez NG. Deciduoid mesothelioma: report of 21 cases with review of the literature. Mod Pathol. 2012;25:1481-95.

16. Huang CC1, Michael CW. Michael (2013) Deciduoid Mesothelioma: Cytologic presentation and diagnostic pitfalls. Diagn Cytopathol 41(7), 629-635.

17. Wolff-Bar M, Dujovny T, Vlodavsky E, Postovsky S, Morgenstern S, Braslavsky D, Nissan A, Steinberg R, Feinmesser M. An 8-year-old child with malignant Deciduoid Mesothelioma of the abdomen: report of a case and review of the literature. Pediatr Dev Pathol. 2015;18(4):327-30.

18. Ushio R, Yamamoto M, Shibata Y, Ishii H, Watanabe K, Takahashi R, Sato T, Kudo M, Miyake A, Kaneko T, Ishigatsubo Y. An autopsy case report of malignant pleural Mesothelioma with Deciduoid features. Intern Med. 2015:54:2915-7.

19. Travis WD, Brambilla E, Burke AP, et al. (2015) WHO classification of tumours of the lung, pleura, thymus and heart. IARC press (Lyon, France) 156-168.

20. Husain AN, Colby T, Ordonez N, Krausz T, Attanoos R, Beasley MB, Borczuk AC, Butnor K, Cagle PT, Chirieac LR, Churg A, Dacic S, Fraire A, Galateau-Salle F, Gibbs A, Gown A, Hammar S, Litzky L, Marchevsky AM, Nicholson AG, Roggli V, Travis WD, Wick M, International Mesothelioma Interest Group. Guidelines for pathologic diagnosis of malignant Mesothelioma : a consensus statement from the international Mesothelioma interest group. Arch Pathol Lab Med. 2009;133:1317-31.

21. Galateau-Salle F, Goubin-Versini I, Duvillard P, Mourra N. Groussard O. Bull de la division française de l'AIP. 2009:49:63-70.
Submit your next manuscript to BioMed Central and we will help you at every step:

- We accept pre-submission inquiries

- Our selector tool helps you to find the most relevant journal

- We provide round the clock customer support

- Convenient online submission

- Thorough peer review

- Inclusion in PubMed and all major indexing services

- Maximum visibility for your research

Submit your manuscript at www.biomedcentral.com/submit
) Biomed Central 